\title{
UNE SCIENCE DES INTELLECTUELS EST-ELLE POSSIBLE?
}

Si la sociologie des intellectuels demeure, au sein de la discipline sociologique, une spécialisation marginale - il suffit de considérer quelques indicateur's simples ${ }^{1}$-, cela tient à une série de facteurs analysables sociologiquement tels que, en particulier, le rapport de la discipline au champ intellectuel et au champ administratif, la hiérarchie des disciplines, les cursus professionnels, etc., tous facteurs qui contribuent à imposer une définition professionnelle des objets dignes d'être traités. En outre, la réticence des saciologues devant ce type d'objet pourrait bien constituer une forme d'autoprotection collective dont le principe est le renoncement mutuel à utiliser des instruments susceptibles de fonctionner comme armes polémiques : contre le terrorisme possible, le silence serait ainsi le meilleur mode de prévention. Enfin, le silence n'apparaît que trop conforme à une représentation positiviste de la science inspirée du modèle prestigieux des sciences exactes avec la distinction entre l'ordre historique de la " découverte" doté d'une valeur purement externe, et l'ordre logique de l'exposé systématique seul jugé digne de la discussion scientifique ${ }^{2}$ : sorte de déontologie professionnelle implicite, l'attitude positiviste conduit à se détourner de toute sociologie des intellectuels. Seule la connaissance des instruments théoriques et méthodologiques mis en œuvre apparait pourtant comme condition de leur utilisation scientifique-

1. Soit les tables des articles publiés dans la Revue française de sociologie de 1960 à 1980 : on peut estimer à 3 sur 601 le nombre d'articles consacrés à ce domaine (dont 2 à la seule école durkheimienne). La revue Actes de la recherche en sciences sociales constitue une exception notable : entre 1975 et 1985 , on peut estimer à 32 sur 313 le nombre d'articles consacrés à la sociologie des intellectuels.

2. Sur les procédés de présentation de soi chez les sociologues, cf. Martin KoHLI, " "Von uns selber schweigen wir ”. Wisssenschaftsgeschichte aus Lebensgeschichten ", in Wolf LEPENIES, Geschichte der Soziologie, Bd'1, Frankfurt am Main, Suhrkamp, 1981, p. 428-465.

Revue de synthèse : IVe S. No 4, octobre-décembre 1986. 
ment contrôlée ; et cette connaissance ne peut être dissociée de ce qui la rend possible, la connaissance historique des agents et des institutions liés à son apparition. Ainsi entendue, la sociologie des intellectuels pourrait être en mesure de remplir une partie des intentions traditionnellement attribuées à la réflexion épistémologique car la réflexion sur les moyens de la connaissance sociologique présuppose l'objectivation du rapport au monde social propre aux producteurs attitrés de discours sur ce monde. Le positivisme d'institution, manifeste dans l'indifférence envers la sociologie des intellectuels, a pour effet d'exposer les sociologues aux pouvoirs incontrôlés des sociologies spontanées et notamment des sociologies spontanées d'intellectuels fondées sur le principe du point de vue privilégié. La connaissance objective du monde social inclut, comme l'une de ses conditions, la connaissance de tout ce qui contribue à imposer, pour les professionnels de la pensée comme pour d'autres, l'univers du pensable : d'où l'attention qui doit ou devrait être portée à l'étude des topiques et des exercices routinisés de l'institution scolaire, à celle des " débats" amplifiés ou suscités par la grande presse intellectuelle (" la fin des maîtres à penser ", "le silence des intellectuels "), etc.

Plus précisément, la sociologie des intellectuels ne peut parvenir à échapper à l'effet de réification qui tend à proposer comme allant de soi des systèmes symboliques qu'à condition de référer toujours ces derniers aux conditions sociales de production et de reproduction des agents qui les produisent et les utilisent. C'est pourquoi la " sociologie de la culture " qui se donne pour objet la réalité préconstruite d'un " esprit du temps " (Zeitgeist) est un obstacle à la connaissance empirique puisqu'elle cumule l'illusion positiviste d'un accès immédiat à la réalité et l'ethnocentrisme intellectuel qui voit dans les "pensées" d'une "époque " l'expression condensée et donc achevée de la réalité sociale $^{3}$. Une approche sociologique de la production intellectuelle actuelle serait donc inconcevable sans l'étude d'une série de transformations objectives telles que, par exemple, les transformations du système scolaire et des relations de ce système au champ intellectuel, les transformations des relations entre le champ universitaire et le champ intellectuel, les transformations du marché des biens symboliques marquées par le brouillage de frontières entre genres, disciplines...

Une sociologie des intellectuels demande seulement qu'on lui concède ce qui, à l'examen, peut paraitre peu de chose : par exemple, que les individus " intellectuels " peuvent faire l'objet de comptages au même titre

3. L'alternative à la réduction brutalement matérialiste aux « infrastructures » a consisté longtemps dans une version « sociologisée » de l'hégélianisme symbolisée par la figure de Georges Lukács. 
que les autres; ou encore, que les catégories de l'entendement sont descriptibles au même titre que des pratiques, ou des institutions, qu'elles peuvent être référées à des contraintes institutionnelles d'utilisation, de transmission, etc.

Les quelques pages qui suivent n'ont pas la prétention de proposer un tableau exhaustif de la sociologie des intellectuels à l'heure présente. Pour prévenir toute méprise, disons d'emblée que le premier souci a été d'éviter tout ce qui pourrait évoquer les effets de palmarès avec ce qu'ils comportent d'injustice, de hâte et de légèreté. À l'énumération de "ce qui se fait" a été préférée une réflexion d'allure volontairement plus didactique sur un ensemble ouvert de principes jugés particulièrement féconds. On a dû, d'autre part, renoncer à évoquer des domaines voisins, en particulier la sociologie de l'art et la sociologie de la science, qui auraient requis des développements spécifiques hors de proportion avec le propos présent.

\section{QUI EST UN INTELLECTUEL?}

Parce que les classements ne sont jamais neutres ou indifférents socialement, il serait hasardeux d'imposer une définition de l'intellectuel, aussi " scientifique " soit-elle, qui ne prenne en considération les usages sociaux d'un terme comportant un enjeu pour les agents : la question de la définition, ou celle des frontières, est d'abord une question pratique enveloppée par des situations classantes apparemment aussi " insignifiantes " qu'une signature de manifeste, de pétition, la collaboration à la presse "intellectuelle", etc. En rupture avec les définitions essentialistes, la description des stratégies d'exclusion et d'inclusion, telle qu'elle est accomplie sur de tout autres domaines, peut procurer une connaissance de ce qu'est un intellectuel en montrant tout ce qui, à un moment donné, doit être fait (dans un sens qui n'a rien de naïvement intentionnel) pour prétendre à ce statut. À côté des stratégies plus ou moins conscientes, celles que les concurrents s'emploient en général à dénoncer, existe tout l'univers des évidences indiscutées communes à tous, dont la thématisation engage de la part du sociologue un travail de dépaysement auquel les intellectuels attitrés n'ont - sauf exception (Karl Kraus) ${ }^{4}-$ aucun intérêt : loin de la

4. Sur les expérimentations de " sociologie-action » réalisées par cet intellectuel viennois sur son milieu d'appartenance, cf. Michael Pollak, « Une sociologie en acte des intellectuels : les combats de Karl Kraus », Actes de la recherche en sciences sociales, 36-37, février-mars 1981 . 
mettre en cause, les luttes internes renforcent l'adhésion primordiale vécue comme foi tacite, qui seule donne sa valeur à l'engagement dans les luttes, avec ce que cela implique d'espoirs, de déceptions, de sacrifices, etc.

On ne peut manquer de référer la définition de l'intellectuel à l'analyse de la constitution de pratiques et d'enjeux spécifiques : l'intellectuel est celui qui est reconnu comme tel par les autres intellectuels dans la mesure où il sait reconńnaître la valeur du seul jeu digne d'être joué par lui. L'aútonomisatión de l'univers intellectuel se manifeste d'abord dans tout un ensemble d'actes de rupture comme l'a montré Pierre Bourdieu au sujet de Flaubert, figure exemplaire, selon lui, de "l'artiste ${ }^{5}$ : le refús de la bêtise bourgeoise, le goût de l'inutile... sont autant de moyens aú service d'une dénégation de la réalité sociale (bourgeoise), vouée à demeurer purement symbolique pour échapper à la platitude, au sérieux, bref à la détermination caractéristique de cette rêalité. L' " artiste » ne se définit pas autrement que par un refus du "boúrgeois ", qui est aussi bien une soumission à l'ordre temporel dominé par lui ${ }^{6}$. Cette mise en évidence de l'ambiguïté fondamentale des transgressions symboliques inhérentes à la condition intellectuelle permet à la sociologie des intellectuels de mener de front l'analyse des conflits entre intellectuels et "bourgeois " et l'analyse des profits de distinction que rapporte à tous l'appropriation des écarts aux règles du bon sens et du bon goût : la dialectique du scandale et de la canonisation qui est contenue dans la logique propre à une économie des biens symboliques, s'accomplit dans la dimension temporelle qu'il faut prendre en compte (par la médiation de l'institution scolaire, de la presse...) pour saisir adéquatement les tránsformations et les dérives continuelles qui affectent l'identité sociale des producteurs et des produits. Le formalisme de « l'art pour l'art », loin de ne représentér qu'une orientation particulière, est donc une tendance à l'œuvre dans toute la logique du fonctionnement d'un univers défini

5. Pierre Bourdiel, "L'invention de la vie d'artiste ", Actes de la recherche en sciences sociales, 2, mars 1975, p. 67-93. Les analyses sur Flaubert - dont l'objet n'était pas essentiellement historique - ne devraient pas conduire à sous-estimer le caractère très long (en France) du processus d'autonomisation d'un "champ intellectuel ». Analysant « la naissance de l'écrivain » au début du Xvıle siècle, Alain Viala s'est emplóyé, en reprenant le cadre des analyses de Pierre Bourdieu, à décrire la mise en place des conditions sociales, et notamment institutionnelles ou quasi institutionnelles (académies, salons, réseaux de clientèle, entreprises de mécénat), d'un « premier champ littéraire » caractérisé par un degré d'autonomie moyen, intermédiaire entre la production hétéronome (pour les grands) et la production autonome (pour les pairs) : cf. Alain VIALA, Naissance de l'écrivain : sociologie de la littérature à l'âge classique, Paris, Minuit, 1985.

6. C'est ainsi que peut être justifiée lidée d'une structuration du champ du pouvoir assignant aux intellectuels le pôle dominé. 
par exclusion de toutes les finalités extérieures. Dans cette logique, une position dominante est celle qui satisfait aux exigences pures d'autonomie, par opposition aux positions basses qui ont partie liée avec l'utilité, le réalisme, les bonnes causes. L'opposition entre le " haut " et le " bas ", qui est inscrite dans les hiérarchies et les luttes pour les subvertir, trouve un renforcement dans l'action de l'institution scolaire qui, à travers les instruments pédagogiques routinisés, dote les sujets les plus accomplis d'un tact indissociablement logique et esthétique qui pousse à rechercher les choses "élevées " " humanité ", " raison ", " sensibilité » - par quoi est " dépassé " ce qui est bas - l' « instinct ", la « passion », la « société » : une analyse de l'inconscient lettré requiert, comme l'avait déjà établi Durkheim dans L'Évolution pédagogique, une histoire sociale de la tradition scolaire nationale. Mais, parce qu'il suppose un ensemble de conditions sociales de possibilité, le formalisme n'est pas un modèle pur anhistorique que les réalités empiriques réaliseraient de façon nécessairement imparfaite : il est plutôt une configuration idéaltypique pouvant être engendrée à partir d'un système général de transformations dont une des variables principales est le degré d'autonomie (ou le degré de dépendance par rapport à d'autres champs). Le poids relatif du public profane cultivé, de l'institution scolaire, du champ politico-administratif... peut produire des effets qui contrecarrent la tendance aux évaluations purement internes (des producteurs sur les producteurs) : ainsi, dans un univers structuré par l'opposition entre le " haut " (l'artiste) et le « bas " (l'écrivain à succès, "bourgeois " ou " populaire »), d'autres principes de classement peuvent apparaître et passer au premier plan. On peut penser aux relations d'opposition suivantes : entre, d'une part, "l'intellectuel total » conforme aux exigences internes, détenteur de surcroît d'un " message " éthique et politique (Sartre $)^{7}$ et, d'autre part, l'écrivain « art pour l'art " récusant tout " message " (Paul Valéry); entre auteurs "doctes " et auteurs "mondains ${ }^{8}$; ou, en régime socialiste, entre l'intellectuel statutairement garanti par la légitimité hétéronome procurée par le Parti et l'intellectuel sans garantie politique voué à témoigner prioritairement de sa conformité, au moins par la réserve ou le silence, pour préserver la part qui peut l'être des exigences internes...

7. Pour une construction sociologique de la trajectoire de cet auteur et de sa définition de l'intellectuel comme principe d'intelligibilité de l'existentialisme, cf. Anna BosCHETT, Sartre et les Temps modernes : une entreprise intellectuelle, Paris, Minuit, 1985, p. 113 et sq.

8. P. Bourdieu, La Distinction, Paris, Minuit, 1979, p. 74-81 ; Norbert Ellas, La Civilisation des moeurs, trad. par Pierre Kamnitzer, Paris, Calmann-Lévy, 1973, p. 11-73. 
Dans un univers social où les systèmes de classement font partie des mécanismes objectifs, l'histoire sociale ne saurait se désintéresser des mots. Ainsi l'apparition en France, à la fin du xixe siècle, du terme d' " intellectuel " mérite l'examen : ni simple étiquette ni expression d'une "prise de conscience " d'un groupe préexistant de tous temps, ce terme est le produit d'une conjoncture marquée par le conflit des modèles d'excellence. Une opération de catégorisation a été accomplie par des écrivains éloignés du monde universitaire sur des universitaires plus ou moins "positivistes" issus de disciplines nouvelles ou de secteurs novateurs de disciplines établies : "l'intellectuel " (ex. : Durkheim) apparaissait, avant tout, au point de vue du bord opposé, comme porteur interchangeable d'une pensée impersonnelle, "abstraite ", " universelle ", et dépourvue, du fait de ses origines basses parfois explicitement soulignées, du sens du sublime (goût, raffinement, fantaisie). Cet usage initial du terme "intellectuel " qui désignait une sous-population très particulière (statistiquement insignifiante) se distingue donc d'usages ultérieurs beaucoup plus souples ou plus flous. Évidemment, il n'y a pas lieu de privilégier en soi l'usage propre à une époque : ce qui importe n'est pas le cheminement d'un mot isolé mais la relation entre des systèmes d'appellations et les structures objectives que ces systèmes contribuent à perpétuer ou à transformer. Vers 1900, "l'intellectuel " était perçu comme une menace pour une production symbolique régie par le modèle du "génie" libre et inspiré". La critique ouvertement politique (de droite) dont "l'intellectuel " était la cible a favorisé depuis lors la méconnaissance de la logique spécifique sous-jacente au travail de classement indigène : "l'intellectuel " était, avant tout, celui qui représentait du point de vue des dominants la position basse associée à l'érudition et à la grisaille... Or, malgré les apparences, cette opposition est loin d'avoir disparu au $\mathrm{XX}^{\mathrm{e}}$ siècle avec l'apparition de " l'intellectuel de gauche ": elle s'est conservée à travers un renversement significatif qui a consisté à associer d'un côté le prophétisme et la gauche et d'un autre côté la routine professorale et le conformisme politique de droite (les " chiens de garde »). Les intellectuels les plus légitimes ont pu bénéficier d'un brouillage des repères à la faveur duquel ils ont pu reproduire les topiques du spiritualisme antiscientifique tout en les parant des prestiges de l'audace théorique et politique ${ }^{10}$. Peut-être n'est-il pas inutile de suggérer à quel point cet inva-

9. L. Pinto, «La vocation de l'universel », Actes de la recherche en sciences sociales, 55 , nov. 1984 , p. 23-32.

10. Sur le cas exemplaire de Sartre, cf. A. Boschetri, op. cit. supra n. 7, p. 153 et sq. 
riant qu'est l'opposition "grand intellectuel "/spécialiste est conforme aux schèmes de pensée scolaires fondés sur la prééminence de ce qui est "original " sur ce qui - justement - est seulement "scolaire": le discours "philosophique" le plus entendu aujourd'hui en France - au point de paraître le seul à être produit ne consiste-t-il pas pour l'essentiel à ressasser " l'irréductibilité " du supérieur (la philosophie) à l'inférieur (la science, les sciences sociales)?

Quelle population (quel corpus) doit donc être délimitée par la sociologie des intellectuels? Étant donné, d'une part, que les « définitions " sont toujours des définitions pratiques mises en œuvre par des agents dans des situations déterminées et, d'autre part, que les opérations de définition tiennent toujours leurs caractéristiques de leurs auteurs, il est instructif de suivre la démarche de Pierre Bourdieu qui, dans une étude d'allure très "empirique ", substitue à la question «qu'est-ce qu'un intellectuel?" la question «qui nomme-t-on ainsi ? " et à celle-ci, enfin, la question ultime " qui détient le pouvoir de nommer ceux qui sont des intellectuels? ». Le " hit-parade des intellectuels français ", publié dans la revue Lire, fournit un instrument privilégié pour l'analyse de mécanismes de sélection qui, habituellement, opèrent de façon diffuse et dispersée ${ }^{11}$. Car l'effet visé d'absolutisation des juges et des jugements n'a de chances d'être aboli que par la vertu de la mise en évidence des caractéristiques sociales du groupe des juges, surtout - comme c'est le cas ici - lorsque le produit final trouve manifestement son principe dans les intérêts attachés à des positions rendues possibles par le brouillage des frontières entre genres intellectuels (romans à succès, essais librement « philosophiques ", journalisme...) et, au travers de celui-ci, par la rencontre entre des trajectoires d'universitaires pour journalistes et de journalistes pour universitaires. La multiplicité des points de vue fait partie de la réalité objective et à ce titre ne saurait être annulée par la vertu d'une "bonne définition". Dans quelle mesure on peut néanmoins éviter de céder au relativisme, c'est ce que l'on tentera plus loin d'élucider.

11. P. Bourdieu, « Le hit-parade des intellectuels français ou qui sera juge de la légitimité des juges? ", in lD., Homo Academicus, Paris, Minuit, 1984, p. 275-286. 
L'UNITÉ D'ANALYSE

Il est hors de propos de présenter ici les implications « théoriques » de la notion de "champ ". Il a paru préférable d'en montrer toute la fécondité à travers les démarches de l'activité de recherche. Ce qui est en cause dans l'utilisation de cet instrument logique est d'abord la question de l'unité d'analyse. L'histoire traditionnelle de la production intellectuelle tend à réaliser une absolutisation de la singularité auteur, texte, école - qui, par nature, détourne de toute interrogation sur la pertinence des propriétés prises en compte et enveloppe une prédilection empiriste pour les données préconstruites du sens commun, proprement dit, ou cultivé (caractérologie spontanée, nomenclatures naïvement utilisées telles que noms de styles, d'écoles...) et de la routine sociologique (" indicateurs" d'âge, d'origine sociale... considérés en soi). Or, de même qu'il serait vain de chercher une définition de "l'artiste " ailleurs que dans l'opposition au " bourgeois", qui en est véritablement constitutive, de même les traits spécifiques des individus reconnus comme " intellectuels " au sein d'un espace relativement autonome ne sauraient se comprendre sans considérer le système des relations qui unissent, d'ailleurs, moins des individus empiriques que l'ensemble des positions objectivement définies occupées par ces individus. Dans le cas analysé par Rémy Ponton ${ }^{12}$ des romanciers de la seconde moitié du XIXe siècle, les propriétés attachées à chacun d'eux apparaissent déterminables seulement à partir d'une opposition fondamentale entre dominants et dominés : les romanciers " psychologues " (Barrès, Bourget...) ont élaboré une esthétique noble par rejet du style jugé vulgaire des auteurs naturalistes (Maupassant, Zola); or l'opposition esthétique interne recouvre et redouble une opposition sous le rapport des origines sociales, de la profession actuelle, etc. Seuls la définition des positions et le découpage méthodique des

12. Rémy Ponton, "Naissance du roman psychologique", Actes de la recherche en sciences sociales, 4, juil. 1975, p. 66-81. Sur la logique des clivages, sous le rapport politique, cf. Christophe Charle, "Champ littéraire et champ du pouvoir : les écrivains et l'affaire Dreyfus », Annales ESC, 2, mars-avr. 1977, p. 240-264; sous le rapport géographique, cf. P. Bourdieu, "La production de la croyance", Actes de la recherche en sciences sociales, 13, févr. 1977, p. 36-37 et C. CHARLE, "Situation spatiale et position sociale, essai de géographie sociale du champ littéraire à la fin du dix-neuvième siècle ", ibid., p. 45-59. 
populations selon l'ordre réglé des emboîtements successifs (écrivains $v s$ bourgeois, romanciers $v s$ poètes, "psychologues " $v s$ naturalistes, etc.) permettent d'engendrer des oppositions significatives et d'établir entre elles des relations d'homologie; et la connaissance systématique des propriétés de position permet de déduire la propension à opérer tel ou tel type de choix (les " psychologues " plutôt attirés par la rive droite, l'ánti-dreyfusisme...) et de saisir la cohérence objective des choix effectués. Ce qui est dit des positions inscrites dans le champ peut être transposé à l'étude des trajectoires : celles-ci, loin d'être données à l'intuition, sont à construire dans l'espace total des trajectoires comparables. Ainsi quand on considère, par exemple, l'univers relativement réglé des carrières académiques, une propriété aussi « objective " que l'âge d'un individu singulier demande à être rapportée à l'âge modal d'accès à certains statuts ou plus précisément à l'ordre modal de la succession des statuts en fonction des âges probables d'accès : la " jeunesse " (universitaire) n'a d'autre définition dans un monde où l'on sait attendre (au point d'en faire une morale) que le temps où l'on n'est pas en état d'occuper la position supérieure détenue par les prédécesseurs.

La prise en compte de données morphologiques permet de compliquer considérablement le modèle d'analyse et d'écarter toute vision simpliste d'un champ structuré par des oppositions univoques et stables. En effet, la reproduction structurale des écarts entre positions hiérarchiquement différenciées paraît aller de soi dans des conjonctures particulières en lesquelles semble garantie la correspondance entre l'état des possibilités objectives et le système socialement conditionné, sinon contrôlé, des aspirations. Dans le champ intellectuel comme en d'autres univers sociaux, une modification des effectifs et, particulièrement, un phénomène d'encombrement, entraînent un certain nombre d'effets obéissant à des mécanismes très généraux. Parmi ceux-ci figurent la stratégie de numerus clausus, évidemment difficile à mettre en œuvre dans des secteurs non réglementés, la stratégie de recours à une " réserve " qui à permis aux disciplines académiques les plus traditionnelles de se perpétuer en recrutant, selon la logique de la " seconde chance", les individus les moins éloignés de la définition conforme... À ces stratégies défensives, il faut ajouter les stratégies d'innovation, pas toujours totalement discernables des précédentes, qui consistent dans une redéfinition des positions par reconversion (ou hybridation) du capital possédé menacé de dévaluation : cas des poètes reconvertis en romanciers "psychologues ", cas des physiologues allemands du XIXe siècle en surnombre qui, inventant la " psychologie expérimentale ", ont investi l'un des terrains traditionnels de 
la discipline philosophique ${ }^{13}$. La création de positions novatrices peut donc en fait recouvrir des réalités très dissemblables allant de la recherche d'une forme nouvelle de légitimité culturelle, comme dans les exemples précédents, à la tentative de ménager un espace flou ayant la vertu d'échapper aux alternatives, comme dans le cas du journalisme intellectuel apparu dans les années $60^{14}$.

Mais en se donnant comme principe d'intelligibilité le "champ ", c'est-à-dire un ensemble complexe et étendu de relations, le sociologue des intellectuels ne s'expose-t-il pas à l'alternative du tout ou rien? En l'absence d'une connaissance exhaustive des déterminations, qui reste bien entendu l'idéal d'une démarche scientifique, un matériel même limité peut procurer le moyen de formuler des hypothèses testables. Il arrive fréquemment que les principales oppositions significatives requises par la construction de l'objet se trouvent présentes à l'état plus ou moins explicite dans le discours intellectuel à travers des marqueurs positifs ou négatifs (" positiviste", " professionnel ", " petit bourgeois ", « allemand »...). Dans son étude consacrée, à partir d'un matériel autobiographique, à l'impuissance intellectuelle de l'écrivain Amiel ${ }^{15}$, Luc Boltanski a entrepris une énumération des divers types de propriétés au moyen desquelles l'identité est construite : ainsi, les oppositions mentionnées par Amiel entre fractions de la classe supérieure, entre sexes, entre traditions nationales, entre modèles d'accomplissement intellectuel... constituent des repères primordiaux à la fois spécifiques et redondants puisqu'en chaque domaine se répète une répulsion envers les pôles extrêmes (commerçant/artiste, homme/femme, écrivain/professeur, Allemand/Français...) qui voue à l'indétermination. Mais le Journal ne saurait être considéré comme un simple "document" sur la recherche d'une identité sociale : il est le moyen par lequel s'accomplit le travail de sublimation qui consiste à transmuer en " œuvre " le ressassement de son indétermination et de l'impossibilité de l'œuvre. On tient ici une belle occasion de récuser toute alternative sommaire opposant l'objectif et le subjectif. Parce qu'une stratégie intellectuelle met toujours en

13. Joseph BeN David, Randall Collins, "Social Factors in the Origins of a New Science : The Case of Psychology ", American Sociological Review, XXXI, 4, août 1966, p. 451-465. La mise en relation des propriétés de structure et des propriétés morphologiques est au centre des nombreux travaux de Victor Karady - impossibles à citer ici dans leur intégralité - consacrés à l'histoire sociale des systèmes universitaires français et hongrois et au centre, bien sûr, de Homo Academicus, op. cit. supra n. 11.

14. L. Pinto, L'Intelligence en action: le Nouvel Observateur, Paris, A. M. Métailié, 1984.

15. Luc Boltanski, "Pouvoir et impuissance : projet intellectuel et sexualité dans le Journal d'Amiel ", Actes de la recherche en sciences sociales, 5-6, nov. 1975, p. 80-108. 
jeu l'image de soi qu'un auteur peut proposer, et d'abord à lui-même, une description rigoureuse de ses stratégies comporte, intimement unies, l'étude des intérêts expressifs associés à une position socialement déterminée et l'étude des profits symboliques procurés par l'opération proprement intellectuelle de mise en forme ${ }^{16}$.

\section{L’ÉCOUTE SOCIOLOGIQUE}

L'analyse des œuvres en tant que telles est contenue dans le projet même de l'approche sociologique qui doit surmonter l'obstacle que constitue pour elle l'opposition entre un point de vue externe (pour biographes, historiens et sociologues) et un point de vue interne (pour lettrés, éventuellement pourvus des ressources modernes de la linguistique, de la sémiologie...). Cas particulier des productions linguistiques, une œuvre déterminée tient ses caractéristiques socialement pertinentes des mécanismes de formation des prix qui régissent le système des relations entre la structure de distribution du capital linguistique et la structure du marché : l'efficacité proprement symbolique, propriété sociale fondamentale par excellence des discours, tient autant à ce qui est dit qu'à une manière de dire ajustée aux exigences imposées par la conjoncture d'élocution et, en particulier, à celles qui découlent de l'appartenance à un champ de production symbolique d'abord défini par son autonomie à l'égard des règles régissant les usages profanes externes. La hiérarchisation immanente à la production des discours des intellectuels exprime donc une élévation croissante du travail de mise en forme : le locuteur savant détient une maîtrise pratique des ressources de thèmes et de modes de traitement d'autant plus éminente qu'il parvient à prendre ses distances envers toute interprétation naïve, naïvement réductrice, de ce qu'il dit. La lecture sociologique d'un philosophe aussi " hermétique " que Heidegger suppose donc de mener conjointement, et avec un soin égal, deux opérations distinctes : par la première, il s'agit de prendre pour objet la correspondance qui s'établit entre le système savant des oppositions heideggériennes pertinentes (" on " « inauthentique "/ " Da-sein " "authentique ") et le système profane des oppositions idéologico-

16. Cf. Sergio MiCEL, Les Intellectuels et le pouvoir au Brésil (1920-1945), Paris, Maison des sciences de l'homme/Presses universitaires de Grenoble, 1981 ; Michaël Pollak, Vienne 1900. Une identité blessée, Paris, Gallimard, 1984 (" Archives ", 14). 
politiques propres à la pensée völkisch de l'Allemagne prénazie (" masses »/ « personne "); par la seconde, est prise en considération la différence interne, proprement philosophique, qui résulte de l'exclusion plus ou moins explicite, des choix alternatifs offerts par l'état du champ à ce moment donné (ici : le néo-kantisme) : ainsi envisagée, l'ontologie heideggérienne peut apparaître comme l'équivalent en philosophie de la "révolution conservatrice " ${ }^{17}$.

Le sens social des discours n'est pas à chercher tant dans ce qui est dit que dans la relation de complicité qui unit dans la reconnaissanceméconnaissance les producteurs et les destinataires de la parole autorisée. Et l'on pourrait aisément transposer à l'analyse de maintes productions philosophiques, comme formes éminentes du discours savant, les principes mis en ouvre pour l'analyse des rites magiques : le contenu manifeste qui se propose à l'activité enchantée de déchiffrement interne des exégètes dissimule les conditions de la croyance, en laissant entendre qu'il y a autre chose à entendre que l'énoncé de la sacralité de la parole et des agents autorisés à la dire ou à l'ouïr (exemple simple : le « concept " de doxa qui, la plupart du temps, reste vide dans la philosophie académique n'a d'autre fonction que de marquer la hiérarchisation savante des discours). On pourrait voir une illustration inattendue (étant donné le poids des traditions intellectuelles) de cette logique dans les analyses consacrées par Marie Ymonet à "l'invention du marxisme en France" : renonçant à la voie traditionnelle de la recherche (inépuisable) du contenu " théorique " " authentique ", elle montre que le destin du " marxisme " n'a pas tenu à ses vertus internes - indiscutées par la posture de sacralisation - mais à des caractéristiques formelles en relation d'affinité avec les intérêts d'individus qui, relativement dépossédés sur le terrain intellectuel et sur le terrain politique, tentaient de gagner, par la référence à une " science " autoproclamée, une double légitimité les mettant à l'abri de toute mise en question soit intellectuelle soit politique ${ }^{18}$.

Démonter les mécanismes de l'efficacité symbolique, cela suppose de prendre en considération le sens vécu par les agents et l'écart à sa vérité objective en tant qu'il est fondé sur des conditions objectives.

17. P. BourdieU, "L'ontologie politique de Martin Heidegger ", Actes de la recherche en sciences sociales, 5-6, nov. 1975, p. 109-156. La méthode mise en ceuvre s'avère également féconde dans le cas de Sartre : cf. A. BoscheTrI, op. cit. supra n. 7, p. 83 et sq.

18. Marie YMONET, « Les héritiers du Capital. L'invention du marxisme en France au lendemain de la Commune ", Actes de la recherche en sciences sociales, 55, nov. 1984, p. 3-14; pour l'analyse d'une forme plus altière de rhétorique (celle du philosophe Etienne Balibar), cf. P. BOURDIEU, « Le discours d'importance. Quelques réflexions sociologiques sur quelques remarques critiques à propos de "Lire le Capital "”, in ID., Ce que parler veut dire, Paris, Fayard, 1982, p. 207-226. 
C'est le contraire même de toute posture objectiviste en sociologie : la connaissance du fait social ne peut être tenue pour achevée que lorsqu'elle est parvenue à inclure la connaissance de la façon dont une société "se paie de la fausse monnaie qu'elle a elle-même fabriquée "; elle impose donc un autocontrôle des conditions de production du discours sociologique (qui ne saurait se confondre avec l'autoassurance procurée une fois pour toutes par les protections académiques statutaires). Concrètement, cela implique d'objectiver les formes indigènes de lucidité - de l'académisme savant à la démystification cynique - qui, à l'œuvre dans le discours sur soi des membres d'un groupe ou d'une institution, ont pour fonction, en proposant une vue " réaliste " des choses, de devancer et, par suite, d'interdire la science des rapports à l'objet et donc la science de l'objet.

Mais le sociologue n'est-il pas, après tout, pris lui-même par une pulsion de "démystification"? Le propos de la démarche sociologique n'est pas de " démasquer " le discours profane derrière les apparences illusoires de l'expression savante; il est de rendre compte des principes de transformation qui règlent la relation entre l'ordre des contenus intellectuels et l'ordre des pulsions sociales. Si la formule de la "sublimation " semble dotée d'un privilège remarquable au point de paraître enfermer à elle seule toute une théorie des intellectuels, ce n'est pas parce qu'elle constituerait le principe exclusif de toute production symbolique mais plutôt parce qu'elle exprime la forme limite d'une tentation ultime découlant des automatismes immanents au champ intellectuel. Analysant les déterminants de la vocation intellectuelle de Freud, en utilisant le matériel empirique autobiographique fourni par L'Interprétation des rêves, Carl Schorske a montré 19 à juste titre le processus de déplacement à la faveur duquel le débat politicofamilial avec le père s'est transformé en un drame purement intérieur : pulsions originelles n'ayant pu être assumées avec leur sens ouvertement social, la relation ambivalente aux puissances établies symbolisées par " Rome" et la tentation de la révolte symbolisée par le personnage d'Annibal n'ont pu trouver la voie de l'expression que dans la forme acceptable de la théorisation psychologique (le "complexe d'Edipe "). La mise en évidence du renoncement aux satisfactions directement temporelles, qui est au principe, d'une part, de la vocation scientifique et, d'autre part, de la retranscription des conflits dans l'intériorité psychique, va certainement à l'encontre de tout le travail individuel puis collectif de transfiguration symbolique accompli par la

19. Carl SCHORSKE, « Politique et parricide dans L'Interprétation des rêves de Freud », in ID., Vienne fin-de-siècle, Paris, Seuil, 1983, p. 177-196. 
psychanalyse : en effet, à travers l'analyse des fantasmes sociaux de Freud, ce qui est en cause n'est rien d'autre que le " fondement transcendantal " (refoulé) de la discipline.

La conquête de cette lucidité conquise sur la psychanalyse risque pourtant de demeurer partielle : elle tend à décrire tacitement comme privation le "choix" de la science au détriment de la politique, consacrant en fait le principe de hiérarchisation lié au point de vue des dominants. La volonté de savoir, trait typiquement " œdipien ", dont rend mal compte la terminologie assez floue de "l'intériorisation ", pourrait après tout être (sous réserve d'analyses empiriques) la forme travaillée que prend le désenchantement envers un idéal du moi qui est d'abord celui du père : même la tentation refoulée de la révolte conserverait encore quelque chose de l'adhésion paternelle au monde social, ne serait-ce qu'une définition tacite de "l'action" (comme opération instrumentale, mesurable à des effets...). Le déplacement du conflit politique vers le conflit intrapsychique qui tend à être considéré comme une simple opération de transposition symbolique (sublimation) peut être vu d'une certaine manière comme l'une des formes que prend le "refus d'hériter" (selon le mot de Pierre Bourdieu au sujet de Flaubert); la défiance envers le père, difficilement assimilable dans le cas présent au repli rêveur sur soi traditionnellement associé à la "sensibilité " intellectuelle (il faut distinguer Freud et, par exemple, Amiel), se retourne en défi, le défi de prendre pour objet de savoir la vie souterraine des familles - largement ignorée d'ailleurs par une sociologie longtemps marquée par le juridisme, l'économisme, etc. La "dépolitisation", au prix de laquelle a été acquise cette connaissance des formes domestiques et intériorisées de domination, a marqué une rupture dans le cela-va-de-soi qui accompagne la transmission dynastique de l'assurance masculine et de la parole officielle (pas nécessairement représentées par la lignée paternelle). La science faite par le fils manifeste l'assomption d'un manque occulté par le père : elle seule - bien mieux que la revanche sociale - a le pouvoir, en " laissant les choses en l'état ", de suspendre la logique de la domination. Le pôle féminin, dont le rôle privilégié dans les modèles intellectuels a été établi par plusieurs auteurs en fonction d'une homologie entre relations entre fractions de la classe dominante et relations entre sexes, détient seul une ressource indispensable dans une situation de conflit avec les "autorités", la force pulsionnelle procurée par la confiance primordiale.

Ces quelques remarques très librement inspirées de la lecture de l'étude exemplaire de Schorske n'ont pas la prétention de traiter un point d'histoire sociale qui renvoie à l'investigation d'un matériel 
empirique. À travers ce « cas " singulier, il a paru intéressant de montrer que, parmi les questions soulevées par la sociologie des discours intellectuels, il y avait d'abord celle de sa propre possibilité. Pour comprendre les présupposés sociaux d'une représentation savante du monde social, le sociologue engage plus ou moins explicitement une théorie de la connaissance du monde social, qui est elle-même solidaire d'une théorie des intellectuels et d'une théorie de la constitution du projet intellectuel laquelle, de son côté, comporte une conception de la construction de l'identité sociale, une conception de la façon dont les oppositions du monde social sont intériorisées par la médiation des structures symboliques transmises par la famille d'origine...

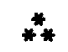

Le projet même d'une connaissance objective suppose de refuser tout privilège épistémologique à une catégorie déterminée d'objets. C'est pourquoi la sociologie du charisme ("don", " génie ") peut apparaître comme un préalable d'une démarche rationnelle fondée sur un postulat d'intelligibilité universelle : elle a une vertu en quelque sorte pédagogique en appliquant à des domaines définis socialement par un statut d'exception un corps de règles méthodiques répétables et généralisables. La sociologie s'inscrit de la sorte dans un processus de laïcisation critique qui tend à neutraliser les effets de l'adhésion doxique à un monde social structuré par l'opposition entre un " haut " et un «bas". Elle implique un relativisme de méthode qui consiste à décrire la pluralité des points de vue éthiques et logiques pris sur le monde sans accorder de prééminence de principe à aucun d'entre eux : dans la mesure où c'est l'opposition même qui fait sens, les descriptions paraissent en principe réversibles par changement du point de vue d'où elles sont effectuées.

L'acte d'objectivation qui s'impose comme exigence, le relativisme de méthode contiennent néanmoins un principe de hiérarchisation. Certes, le point de vue d'où est obtenue la connaissance du système des positions est une position particulière justiciable d'une analyse : on peut montrer que l'accumulation des savoirs en certaines positions du champ intellectuel et au sein du champ scientifique est l'un des effets qui découlent de la logique des "mécanismes " objectifs de concurrence entre positions, que le principe de cette concurrence est constitué par une forme spécifique de capital symbolique, instrument et enjeu de lutte... Il reste que l'objectivation s'accomplit contre la logique du champ intellectuel qu'elle vise précisément à manifester en transgressant les règles de solidarité tacitement liées à l'appartenance : 
la condition (et le prix) du savoir est le renoncement à certains profits de l'appartenance et notamment aux plus subtils d'entre eux, ceux que procure la croyance heureuse. La science de la croyänce suppose une posture intellectuelle qui enferme à l'étát pratíque une éthique.

Deux tentations hantent toute theorie de la connaissance, comme on l'a suggéré plus haut au sujet de Freud : celle d'une vision idéaliste posant un pur esprit cómme sujet de la connaissance; celle d'une vision réaliste concevánt la connaissance comme moyen de satisfaire directement ou indirectement des intérêts particuliers. Pour échapper à pareille alternative il faut supposer la possibilité d'un intérêt socialement conditionné qui est tel que la connaissance objective, et seulement elle, apparaît en mesure d'intéresser le sujet de cette connaissance : l'objectivation n'est pas un pur accroissement d'information mais une autoobjectivation qui enferme une transformation de ce śujet. C'est dans la psychanalyse que l'on a pu reconnaître d'abord mais doit-elle en garder le monopole ? - le projet de faire coïncider l'intérêt théorique de connaíssance et l'intérêt pratique de libération : comme l'a montré Jürgen Habermas ${ }^{20}$, cette coïncidence est un principe d'autoréflexion devant être considéré à la fois comme résultat et comme présupposé de l'acte de connaissance. On comprend que l'éthique 'soit présente seulement à l'état pratique dans cet acte puisqu'en voulant l'autonomiser - le sociologue prenant la parole pour dire la loi - on aboutirait paradoxalement à perturber la logique propre de la connaissance, et donc, par suite, à compromettre la logique même de la libération.

Louis Pinto, C.N.R.S.

20. Jürgen Habermas, Connaissance et Intérêt, Paris, Gallimard, 1979 ( $\mathrm{Tel} »)$, p. 247-331. On trouvera une certaine convergence sur ce point avec un texte de $P$. BourDIEU tel que Leçon sur la leçon, Paris, Minuit, 1982. 